\title{
The Games between Central and Local Governments in the Newest Regulatory Policy of the Real Estate Market
}

\author{
Ming-li Gong ${ }^{1}$, Han Jiang ${ }^{2}$ \\ ${ }^{1}$ Department of Mathematics, Jinan University, Guangzhou, Guangdong Province, China \\ ${ }^{2}$ School of Economics, Renmin University, Beijing, China \\ sherogml@foxmail.com danielblack@foxmail.com
}

\begin{abstract}
Chinese central government has promulgated a series of policies and measures to regulate the real estate market to restrain the rapid increasing of the house price, but the effect seems unsatisfactory. This paper mainly aims at analyzing the origin of this issue by the game theory, thus we establish an Intergovernmental Static Nash Equilibrium model and a Mixed Strategy Nash Equilibrium Model as well. Based on the final equilibrium point, we find that it's the uneven interests' distribution between central and local government which leads to the failure of the newest policy, and strengthening regulation and adjusting the structure of interests' distribution can be efficient ways to solve the problem.
\end{abstract}

Key Terms - real estate market ; government ; mixed strategy Nash equilibrium model.

\section{I . Introduction}

With the coming out of the newest regulatory policy from the Chinese Central Government on February 20, 2013, a new round of tightening real estate regulation has begun. Since March 2013, Beijing, Shanghai, Chongqing, Xiamen, and many other cities has launched the implementing rules. Compared with Beijing or Shanghai whose regulations are detailed and specified, Guangzhou and Hefei's rules seem very indistinct. For instance, 20\% capital gains tax on house sales , real estate tax, down-payment and restriction on the purchases of second house aren't mentioned. Therefore, the game between the central and local governments become the most popular topic among academic circles.

Real estate market regulation involves games of multiparty interests, and the game between the central and local governments is the most significant one. Based on game theory, this paper combines Nash equilibrium model under static conditions with dynamic Nash equilibrium model to analyze the origin of the problem, and try to find out solutions that really work.

\section{II . Intergovernmental Static Nash Equilibrium Model}

For the central government, its economic goal aims at obtaining more supports from voters, and thus getting more prestige for itself. Therefore, its policies are always made so as to expand the national wealth and keep the harmony of society. ${ }^{[1]}$ However, local governments' targets are different and various. They are not only chasing for the increase of local people's wealth, but also maximizing their own interests. When object of both sides is not the same, here comes the policy game. Based on this fact, our article established a static Nash equilibrium model to analyze the situation.

\section{A) Assumptions}

1. Both the central and local government are homooeconomicus in economic sense, and pursue the maximum utility of themselves;

2. Both the central and local government are risk neutral;

3. The central government won't change the current policy until they find the aberrant behavior of the local government;

4. The local government has natural advantages over the information of local property market, which makes the supervision from central government isn't efficient enough to find and stop the aberrant behavior of the local government;

5. The parameter describing the economic development level of the central government in the utility function in this model depends on the average level of the national economy.

\section{B ) Parameters}

So as to present the model clearly, a series of parameters are introduced as follows.

$I_{c}$ describes the max revenue of the central government, and $I_{c}{ }^{\prime}$ describes the revenue when the local government doesn't comply with the policy, $I_{c}>I_{c}{ }^{\prime} ; I_{l}$ represents the maximum revenue of the local government while $I_{l}{ }^{\prime}$ is for the case that it violates the rule, $I_{l}{ }^{\prime}>I_{l} ; C_{0}$ is the cost of the central government for supervision; $C_{1}$ is the cost for local government to carry out the policy, and $C_{2}$ is the cost when violating the rules, $C_{1}>C_{2}$.

C) Model Formulation

Based on the parameters above, the Intergovernmental Static Nash Equilibrium Model is established as follows:

\begin{tabular}{|c|c|c|c|}
\hline \multicolumn{4}{|c|}{ Local government } \\
\hline \multirow{4}{*}{$\begin{array}{c}\text { Central } \\
\text { government }\end{array}$} & Supervision & $\left(I_{c}-C_{0}, I_{l}-C_{1}\right)$ & $\left(I_{c}{ }^{\prime}-C_{0}, I_{l}{ }^{\prime}-C_{2}\right)$ \\
\cline { 2 - 4 } & Non-supervision & $\left(I_{c}, I_{l}-C_{1}\right)$ & $\left(I_{c}{ }^{\prime}, I_{l}{ }^{\prime}-C_{2}\right)$ \\
\hline
\end{tabular}


According to the Nash equilibrium matrix, it can be inferred that, once the central government takes measures to supervise the local government, the revenue for the latter is $I_{l}-C_{1}$ if the local carries out the policy and it's $I_{l}{ }^{\prime}-C_{2}$ when the local doesn't do so. As the revenue in case 2 is more than that of case 1 , the local government is surely not to observe the central government's instructions. Similarly, if the central government does nothing, the local government will still choose to violate.

Going the other way, once the local government follows the current policy, the central government is prone to give up the supervision over the local government as it can get a revenue of $I_{c}$ which is more than the value $I_{c}-C_{0}$ that it earns when taking measures. Furthermore, this choice of central government also applies to the case when the local government doesn't carry out the policy.

All in all, the supervision carried out by the central government doesn't perform good enough to improve the situation of the two in the game. The current equilibrium of non-supervision and non-compliance isn't the best case, as it's of high possibility that the local government will hope to see the rise of the housing prices in order to increase its revenue, worse still, the regulatory policies of the central government is under the threat of losing efficacy.

\section{III . Dynamic Mixed Strategy Nash Equilibrium Model}

\section{A) Assumptions}

The first model in the paper has made a discussion over the game between the central and the local governments from the basic level, and got the equilibrium point where neither the central nor the local government shall take positive acts. ${ }^{[2]}$ However, it's not the best case to some extent. So as to analyze the game further more, it calls for the following Dynamic Mixed Strategy Nash Equilibrium Model, and some essential assumptions are listed below.

VI. All the functions involved in this model are linear ones;

VII. The newest five policies on the real estate market are set as policy $X$, and its revenue is supposed to be calculated with the utility coefficient $k(k \geq 1)$ once functioned.

\section{B) Parameters}

$a$ : The benefit proportion of the central government in condition that the local government complies with policy $X$, while the local government shares the left $1-a, a \in(0,1)$;

$b$ : The benefit proportion of the extra revenue for the local government once it does not observe the newest policy, and the remaining $1-b$ belongs to the central government, $b \in(0,1)$;

$\varepsilon$ : The degree of violation of the local government, and the bigger $\varepsilon$ is, the more severe the case gets to be;

$A$ : The cost for the central government to supervise on the local government; and the latter must pay a penalty with $M$ times of that value once found to violate;
$U c_{i}: i=1,2,3,4$, the revenue of the central government in case $i$

$U l_{i}: i=1,2,3,4$, the revenue of the local government in case $i$;

$p:$ The probability of the violation for the local government;

$q$ : The probability of the supervision for the central government;

$U c$ : The expected revenue of the central government;

$U l$ : The expected revenue of the local government.

\section{C) Model Formulation}

With the parameters and assumptions all above, four cases are taken into consideration as follows:

1. The local government complies with policy $X$ while the central government takes a supervision

$$
U c_{1}=a k X-A, U l_{1}=(1-a) k X
$$

2. The local government complies with policy $X$ while the central government doesn't supervise it

$$
U c_{2}=a k X, U l_{2}=(1-a) k X
$$

3. The local government violates policy $X$ while the central government doesn't supervise it

$$
\begin{aligned}
& U c_{3}=(1-\varepsilon) a k X-b X \varepsilon \\
& U l_{3}=(1-a)(1-\varepsilon) k X+b X \varepsilon
\end{aligned}
$$

4. The local government violates policy $X$ while the central government supervises it

$$
\begin{aligned}
U c_{4} & =a k X(1-\varepsilon)+M b X \varepsilon-b X \varepsilon-A \\
& =(1-\varepsilon) a k X+(M-1) b X \varepsilon-A \\
U l_{4} & =(1-a) k X(1-\varepsilon)+b X \varepsilon-M b X \varepsilon \\
& =(1-a)(1-\varepsilon) k X-(M-1) b X \varepsilon
\end{aligned}
$$

Taking the real situation into consideration, the local government has natural advantages over the information on local property market, which causes that the supervision from central government isn't efficient enough to find and stop the aberrant behavior of the local government ${ }^{[3]}$; here a new parameter $\mu$ is introduced in to describe the probability for the central government to find the violations of the local government, along with a new case for the game.

5. The local government violates policy $X$ while the central government supervises and finds its violation .

$$
\begin{aligned}
U c_{5} & =a k X(1-\varepsilon)-b X \varepsilon-A \\
& =(1-\varepsilon) a k X-b X \varepsilon-A \\
U l_{5} & =(1-a) k X(1-\varepsilon)+b X \varepsilon
\end{aligned}
$$




$$
=(1-a)(1-\varepsilon) k X
$$

\section{D) Model Analysis}

On the basis of the analysis above as well as the probability for the governments to take different measures, the expected revenue of the governments under policy $X$ can be calculated as follows.

$$
\begin{aligned}
U c= & (1-p) q U c_{1}+(1-p)(1-q) U c_{2} \\
& +p(1-q) U c_{3}+p q \mu U c_{4}+p q(1-\mu) U c_{5} \\
U l= & (1-p) q U l_{1}+(1-p)(1-q) U l_{2} \\
& +p(1-q) U l_{3}+p q \mu U l_{4}+p q(1-\mu) U l_{5}
\end{aligned}
$$

On the purpose of probing into the relationship between the maximum revenue of the central government and its regulation, we solved the first derivative of $U_{c}$ to $q$, and the result is shown below.

$$
\begin{aligned}
\partial U c / \partial q= & -p U c_{1}-(1-p) U c_{2}-p U c_{3} \\
& +p \mu U c_{4}+p(1-\mu) U c_{5}
\end{aligned}
$$

Taking the benefit function under each case into the equation, the expression can be written like this:

$$
\partial U c / \partial q=p \mu M b X \varepsilon-A
$$

Set the value of the equation to be zero, we can get the value like equation (9).

$$
p^{*}=A / \mu M b X \varepsilon
$$

Similarly, solving the first derivative of $U l$ to $p$, the expression comes to be equation (11) when it equals to zero.

$$
\begin{aligned}
& \partial U l / \partial p=b \varepsilon X-b(1-a) k X-q \mu M b X \varepsilon \\
& q^{*}=[\varepsilon X-(1-a) k X] / \mu M X \varepsilon
\end{aligned}
$$

In summary, the Dynamic Mixed Strategy Nash Equilibrium Model finally becomes steady with the values of $p$ and $q$ shown in equation (12).

$$
\begin{aligned}
p^{*} & =A / \mu M b X \varepsilon \\
q^{*} & =[\varepsilon X-(1-a) k X] / \mu M X \varepsilon
\end{aligned}
$$

As it can be seen by the equilibrium solution, the probability of violation of local government is proportional to the central government's regulatory costs, while it's inversely related to the penalty for its irregularity. Consequently, the revenue of central government shall decrease along with the rising cost for supervision, hence, the central government is prone to let up regulation and the local government is more likely to break the policy so as to raise their revenue; this however, coincides with the analysis of the first model. On the other hand, enhancing the fine proportion of local government may reduce the probability for the local government to violate. Thus, reducing the regulatory costs of the central government and increasing the punishment on the non-compliance of local government seem to be two efficient measures to lower the probability of non-compliance of local government.

Furthermore, equatio(12) also indicates that $p^{*}, q^{*}$ are two inverse functions of $\mu$, this means that the local government will conscientiously implement the policies to evade the risk once the central government improves its regulatory efficiency; as long as the local government maintains the status quo, the probability of the central government's regulation shall drop down which helps to raise its revenue and ensures strict implementation of the central government's policy.

\section{IV . Conclusion}

As it can be seen from above, local government's main objective is maximizing its own utility without damaging the interests of central government obviously .Local government takes full advantage to get as much benefit as they can at the expense of passive implementation of central government policies. Finally, the separation of regional interests and central regulation will eventually threaten the interests of whole society. Therefore, it is only to strengthen regulation and adjust the structure of interests' distribution, which can fundamentally solve the problem of real estate regulation.

\section{Acknowledgements}

Here and now, I would like to extend my sincere thanks to all those who have helped me with the paper. Meanwhile, I shall say thank you to the ICASSR 2013 conference which has provided such a wonderful platform for us to show ourselves. Thank you very much.

\section{References}

[1] JIAN Xiao-bin et al,'Local Government Regulation of the Multiple Gamble in Real Estate",Territory \& Natural Resources Study,2011

[2] Zhou Jian-jun,Dai Zhi-xiang,"the Game between Central and Local Governments on the Regulation of the Real Estate Market",The Theory and Practice of Finance and Economics, 2012

[3] Tadj L, Touzene A."A QBD approach to evolutionary game theory". Appl. Math. Modeling . 2003

[4] Fan Qiaoyan ,'Multi-player Game and Financial Risk in Real Estate Market", South China Finance, 2009

[5] MU Ling-ling,LI Pei,CHEN Li-wen , "Sequential Equilibrium of Real Estate Game Model Based on Asymmetric Information Theory",Systems Engineering , 2010 